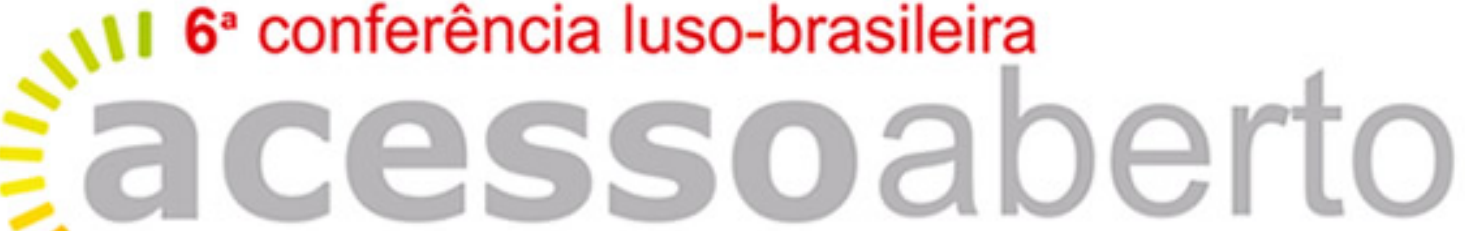 "IIII}

INÍCIO SOBRE ACESSO ÁREA PESSOAL PESQUiSA CONFERÊNCIAS ACTUAIS NOTíciAs UFBA ALOJAMENTO GUIA SIMPLIFICADO DE SALVADOR INSCRIÇÕES PROGRAMAÇÃO

Início > 6a Conferência Luso-Brasileira sobre Acesso Aberto > 6a Conferência Luso-Brasileira sobre Acesso Aberto > Programa

ROGRAMAÇÃo

\begin{tabular}{|c|c|}
\hline $4 \mathrm{de} O \mathrm{~L}$ & ia da UFBA \\
\hline $19 \mathrm{H}-21 \mathrm{H} 00$ & $\begin{array}{c}\text { Sessão de Abertura } \\
\text { Paulo Miguez } \\
\text { (Vice-Reitor da Universidade Federal da Bahia) } \\
\text { Bianca Amaro } \\
\text { (Representante do IBICT) } \\
\text { Rui Vieira de Castro (Vice-Reitor da Universidade do Minho) } \\
\text { poão Nuno Ferreira (Vogal do Conselho Diretivo da Fundação para a Ciência e Tecnologia) } \\
\text { Lidia Maria Batista Brandão Toutain - Superintende do Sistema de Bibliotecas da UFBA } \\
\text { Hildenise Ferreira Novo - Diretora do Instituto de Ciência da Informação } \\
\text { Apresentação Musical } \\
\text { Grupo de Percussão da UFBA } \\
\text { Confraternização }\end{array}$ \\
\hline
\end{tabular}

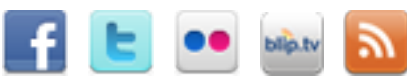

SISTEMA ELETRÓNICO DE

ADMINISTRACÃO D

CONFERÊNCIAS

Ajuda

UTILIZADOR

Nome utilizador ClarissePais

Senha $\quad$..........

$\square$ Memorizar login e senha

Acesso

CONTEÚDO DA CONFERÊNCIA Pesquisa

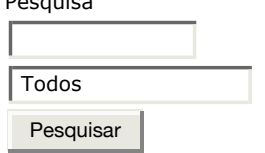

Pesquisar

Informações sobre a Conferência

" Visão Geral

》 $\frac{\text { Programa }}{\text { Inscrições }}$

» Organização e Parceiros

$\gg$ Cronograma

Navegar

- Por Conferência

- Por Autor

TAMANHO DA FONTE

A $\mathrm{A}$

INFORMAÇÃO

- Para Leitores 
de Castro, Monica da Silva Auler, Rosângela Cordeiro de Souza Assef Neto, Simone Pereira

Lermontovf, Vanessa Suane de Souza, Paulo Eduardo Potyguara Coutinho-Marques, Maria Cristina

Soares Guimarães, Cícera Henrique da Silva, Michelle Franzelotti.

SERÃO AS POLÍTICAS INSTITUCIONAIS MANDATÓRIAS, ASSIM TÃO MANDATÓRIAS? QUAL O GRAU DE CUMPRIMENTO? O CASO DA BIBLIOTECA DIGITAL DO IPB

Autora: Clarisse do Céu Pais, Paula Odete Fernandes, José António Sequeira Capela France e Sérgio lexandre Carvalho da Costa

METADADOS E REPOSITÓRIOS INSTITUCIONAIS: UMA RELAÇÃO INDISSOCIÁVEL PARA A

QUALIDADE DA RECUPERAÇÃO E VISIBILIDADE DA INFORMAÇÃO

Autoras: Caterina Groposo Pavão, Janise Silva Borges da Costa, Manuela Klanovicz Ferreira, Zaida Horowitz

REPOSITÓRIO INSTITUCIONAL DA UNIVERSIDADE FEDERAL DA BAHIA COMO MECANISMO DE

ISIBILIDADE PARA OS PROGRAMAS DE PÓSGRADUAÇÃO

Autores: Susane Barros, Flávia Rosa, Rodrigo França Meirelles

\begin{tabular}{|c|c|}
\hline $15 \mathrm{H} 30-16 \mathrm{H} 00$ & Coffee Break \\
\hline $16 \mathrm{H} 0-17 \mathrm{H} 00$ & $\begin{array}{l}\text { Sessão } 4 \text { - O meu poster num minuto... } \\
\text { A AMPLA DISSEMINAÇÃO DOS RESULTADOS DE PESQUISAS CIENTÍFICAS BRASILEIRAS POR MEIO } \\
\text { DO OASISBR } \\
\text { Autores: Bianca Amaro, Washington Ribeiro, Tainá Assis, Alan Oliveira, Teila Carvalho } \\
\text { A IMPORTÂNCIA DO DEPÓSITO DE PUBLICAÇÕES ATRAVÉS DA INTEROPERABILIDADE COM } \\
\text { OUTROS SISTEMAS DE INFORMAÇÃO: O CASO DO REPOSITÓRIUM } \\
\text { Autor: Ricardo Saraiva } \\
\text { ADVOCACY COMO ESTRATÉGIA PARA CONSOLIDAÇÃO DAS INICIATIVAS DE ACESSO LIVRE À } \\
\text { LITERATURA TÉCNICO CIENTÍFICA } \\
\text { Autor: Renato Reis Nunes } \\
\text { ARQUIVO AUDIOVISUAL DA UNIVERSIDADE ABERTA: RECURSOS EDUCACIONAIS EM ACESSO } \\
\text { ABERTO } \\
\text { Autora: Madalena Carvalho } \\
\text { AVALIAÇÃO DO NIVEL DE PERCEPÇÃO E ADERENCIA DA COMUNIDADE ACADEMICA DA UEM }\end{array}$ \\
\hline
\end{tabular}




\title{
SERÃO AS POLÍTICAS INSTITUCIONAIS MANDATÓRIAS, ASSIM TÃO MANDATÓRIAS? QUAL O GRAU DE CUMPRIMENTO? O CASO DA BIBLIOTECA DIGITAL DO IPB
}

\author{
Clarisse Pais. clarisse@ipb.pt \\ Paula Odete Fernandes. pof@ipb.pt \\ José António Sequeira Capela France.zeto@ipb.pt \\ Sérgio Alexandre Carvalho da Costa. a27163@alunos.ipb.pt \\ Instituto Politécnico de Bragança
}

Palavras-chave: Biblioteca Digital do IPB; Repositório institucional; Monitorização; Política mandatória.

\section{Introdução}

Em 30 de abril de 2010, o Conselho Permanente do Instituto Politécnico de Bragança (IPB), aprovou por unanimidade a Política de Auto-Arquivo de Publicações na Biblioteca Digital do IPB ${ }^{1}$, tornando-se na primeira instituição portuguesa de ensino superior politécnico a possuir uma política de obrigatoriedade de autoarquivo de todas as publicações produzidas pelos seus docentes e investigadores. Posteriormente, em 10 de Janeiro de 2011, foi publicado o Regulamento n. ${ }^{\circ} 14 / 2011^{2}$ - Regulamento do Sistema de Avaliação do Desempenho do Pessoal Docente do Instituto Politécnico de Bragança (RAD) - que veio reforçar a obrigatoriedade do autoarquivo, impondo o depósito prévio da produção científica na Biblioteca Digital do IPB (adiante designada por repositório), como condição para ser considerada na avaliação de desempenho. De facto, este regulamento estabelece que "São apenas considerados os artigos depositados na Biblioteca Digital do IPB”. Desta forma, o repositório do IPB foi o primeiro repositório em instituições do ensino superior a ser interoperável com o sistema institucional de avaliação docente (RAD) (Pais, Clarisse; Alves, Albano, 2013).

Para que haja uma ligação ao RAD, os docentes são obrigados a indicar os handles dos registos correspondentes às suas publicações depositadas no repositório do IPB. Com base no handle, são descarregados do repositório os metadados da publicação (título, ano, autores, URI), sendo logo de seguida apresentada a valoração respetiva (Pais, Clarisse; Alves, Albano, 2013).

Desde a promulgação da política, já decorreram três ciclos de avaliação de docentes (2005-2007, 2008-2010 e 2011-2013) e portanto, neste trabalho pretende-se fazer uma análise comparativa entre as publicações depositadas no repositório e as publicações com afiliação do IPB referenciadas na Web of Science e na Scopus, inferindo ainda se essas publicações depositadas no repositório estão em Acesso Aberto.

\section{Metodologia}

A metodologia utilizada consistiu em retirar os dados relativos ao Instituto Politécnico de Bragança na WOS, na Scopus e os existentes no repositório. Todos esses dados foram utilizados para criar tabelas dinâmicas - no excel - que permitem cruzar dados e combinar valores de diferentes campos. Este estudo de monitorização também permitiu verificar a consistência dos metadados no repositório, verificar se as publicações que têm mais citações na WOS e na Scopus estão em acesso aberto no repositório.

\footnotetext{
${ }^{1}$ http://www.ipb.pt/go/g332

2 http://www.ipb.pt/go/d391
} 


\section{Resultados preliminares}

Nos três ciclos de avaliação, com datas limite de depósito, deliberadas pelo Conselho Técnico-Científico do IPB, verificou-se um acréscimo de depósitos no repositório superior a $93 \%$.

Quando se faz uma análise temporal referente aos três ciclos de avaliação (2005-2007, 2008-2010 e 2011-2013) verifica-se que a percentagem da taxa de depósitos ultrapassa os $86 \%$, de documentos referenciados na WOS e na Scopus. No entanto, os documentos não depositados referenciados na WOS e na Scopus, alcançou os $14 \%$.

Nos três ciclos de avaliação foi verificado que $79 \%$ dos documentos ficaram em Acesso Aberto e $21 \%$ em acesso restrito. No entanto, do primeiro ciclo para o último ciclo verificou-se um aumento do acesso restrito de $18 \%$ para $37 \%$, respetivamente.

Relativamente às publicações mais citadas na WOS e Scopus, analisaram-se todos artigos que receberam até 10 citações. Verificou-se que $67 \%$ dos artigos estão em Acesso Aberto e 33\% em acesso restrito.

\section{Conclusão}

Como foi o primeiro Politécnico em Portugal a implementar uma política de autoarquivo obrigatória, o relatório efetuado pelo Projeto PASTEUR4OA ${ }^{3}$, coloca o IPB em segundo lugar, a nível mundial, em termos da taxa de depósitos dos artigos nos universos dos repositórios com políticas obrigatórias.

No caso da Biblioteca Digital do IPB, verifica-se que desde da implementação da política de autoarquivo, tem sido parcialmente cumprida porque está associada ao Sistema de Avaliação do Desempenho do Pessoal Docente do Instituto Politécnico de Bragança.

A avaliação dos dados, permitiu aferir que as políticas de obrigatoriedade institucionais não são $100 \%$ eficazes e portanto terão que se incorporar nos repositórios procedimentos que possibilitem a interoperabilidade com outros sistemas e serviços, como por exemplo, o CrossRef Metadata Search, ou API's da WOS e da Scopus.

Mesmo lutando contra estas particularidades, há docentes, para quem o repositório é fundamental e depositam toda a produção científica com regularidade. As potencialidades que esta plataforma possui, são indiscutíveis em relação a muitas outras plataformas, porque para além de todas as publicações ficarem integradas, também o curriculum DeGóis é imediatamente atualizado.

Referências Bibliográficas

FCT - Politica sobre Acesso Aberto a Publicações Científicas resultantes de Projetos de I\&D Financiados pela FCT. [Em linha]. 2014. [Consult. 29 jun. 2015]. Disponível em WWW: https://www.fct.pt/documentos/PoliticaAcessoAberto_Publicacoes.pdf PAIS, Clarisse; ALVES, Albano - Biblioteca Digital do IPB: integração, partilha e acesso aberto. In Rodrigues, Eloy; Swan, Alma; Baptista, Ana Alice (Eds.) Uma Década de Acesso Aberto na UMinho e no Mundo [Em linha]. Braga : Universidade do Minho, Serviços de Documentação, 2013. [Consult. 29 jun. 2015]. Disponível em WWW: http://hdl.handle.net/10198/8982. ISBN 978-989-98704-0-6.

PASTEUR4OA - Working Together to Promote Open Access Policy Alignment in Europe - Synthesis Report. Work Package 3 report: Open Access Policies [Em linha]. 2015. [Consult. 29 jun. 2015]. Disponível em WWW: http://bit.ly/1zrJd8y

\footnotetext{
${ }^{3}$ http://ec.europa.eu/research/participants/data/ref/h2020/grants manual/hi/oa pilot/h2020-hi-oa-pilotguide en.pdf
} 